\title{
Chemokines and atherosclerosis: what Adam Smith has to say about vascular disease
}

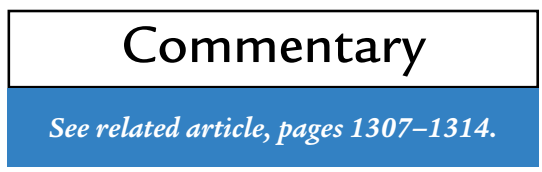

\author{
Barrett J. Rollins \\ Department of Adult Oncology, Dana-Farber Cancer Institute, Department of Medicine, Brigham and Women's Hospital, \\ Harvard Medical School, Boston, Massachusetts, USA \\ Address correspondence to: Barrett J. Rollins, Dana-Farber Cancer Institute, 44 Binney Street, Boston, Massachusetts, USA. \\ Phone: (617) 632-3896; Fax: (617) 632-5998; E-mail: barrett_rollins@dfci.harvard.edu.
}

J. Clin. Invest. 108:1269-1271 (2001). DOI:10.1172/JCI200114273.

\begin{abstract}
"The division of labor, so far as it can be introduced, occasions, in every art, a proportionable increase of the productive powers of labor.”

Adam Smith, An Inquiry into the Nature and Causes of the Wealth of Nations, 1776
\end{abstract}

At the dawn of the industrial age, the social philosopher and economist Adam Smith wanted to explain how some countries were able to create what he called the "opulence" that other countries lacked. Smith suggested that the foremost driver of wealth was the division of labor because it directly results in increased productivity. In his famous example, one worker might make 20 pins a day, but when 10 workers divide the various tasks required to make a pin, they might make 48,000 a day, or an average of 4800 pins per worker per day. This concept is so powerful that it would be surprising if natural selection did not reward biological systems that do the same thing.

To pursue this idea, one might, by straining credulity right to the breaking point, draw an analogy between inflammation and manufacturing. The "product" is effector cell infiltration into target tissues, which is a complex, multistep process involving alterations in cell adhesion, motility, and gene expression. The "workers" are chemokines, an equally complex family of 50 or so small secreted proteins that are chemoattractants for the leukocytes that make up the infiltrate (1). Here, then, is a system that seems tailor-made for the benefits of division of labor, and recent work on the function of chemokines in disease suggests that this is exactly what has happened.

\section{Chemokines and atherosclerosis} One of the clearest examples of the essential role of chemokines in pathobiology involves atherosclerosis. In broad strokes, the inflammatory model of atherogenesis (2) suggests that insults to endothelial or smooth muscle cells, such as hypercholesterolemia or flow shear stress, stimulate the production of leukocyte chemoattractants that are both displayed on the luminal surface of endothelial cells and also secreted into the subendothelium. When these factors activate their receptors on rolling leukocytes, this induces firm integrindependent adhesion to the endothelium, followed by diapedesis into the subendothelium. Among the most important of the migrating cells in this model are monocytes, which differentiate in situ into macrophages and take up cholesterol to become the foam cells of the fatty streak. The target cell specificity of the aptly named monocyte chemoattractant protein-1 (MCP-1) makes this chemokine a superb candidate for the signal that brings circulating monocytes into the vessel wall; MCP-1 attracts monocytes but not neutrophils, and it stimulates the adhesion of monocytes to endothelial cells (3). Mice rendered genetically deficient for MCP- 1 or its receptor, CCR2, are protected from vascular lesions in several atherosclerosis models (4-7).

With such a tidy pathogenetic model available, perhaps it was understandable that many in the field have been disinclined to recognize the significance of studies implicating CXCR2 in atherogenesis. CXCR2 is the receptor for IL-8 and other CXC chemokines that have the three-amino acid gluta- mate-leucine-arginine (ELR) motif near their N-termini. These chemokines are potent chemoattractants for neutrophils and not monocytes, the converse of MCP-1. Nonetheless, the relevance of CXCR2 to atherogenesis was demonstrated in a complicated but illuminating study in which atherosclerosis-prone mice were lethally irradiated and reconstituted with bone marrow donated by wild-type or CXCR2 knockout mice (8). In the chimeras made with CXCR2-/- bone marrow, hematopoietic cells (including, of course, monocytes) were CXCR2-deficient, and these mice had significantly less vascular disease than chimeras made with $C X C R 2^{+/+}$ bone marrow. The paradox posed by this study is that although CXCR2 is important for neutrophil chemoattraction, neutrophils are absent from this atherosclerosis model. What is CXCR2 doing here?

\section{The unexpected role of CXCR2 in the monocyte}

In retrospect, one notes early reports indicating that CXCR2 is expressed by monocytes (9, 10). Furthermore, despite IL-8's inactivity in monocyte chemotaxis assays, this factor induces a calcium flux and respiratory burst in monocytes (11) and enhances adhesion of monocytes in static assays (12). Another ELR-containing CXC chemokine, GRO- $\alpha$, similarly enhances adhesion to stimulated endothelial cells (13). More recently, Gerszten et al. elegantly demonstrated IL-8's ability to induce firm arrest of monocytes on endothelial cells under conditions of physiological flow (14). These results strongly suggest that CXCR2 and one or more of its ligands play a role in atherosclerosis. Even so, according to the 


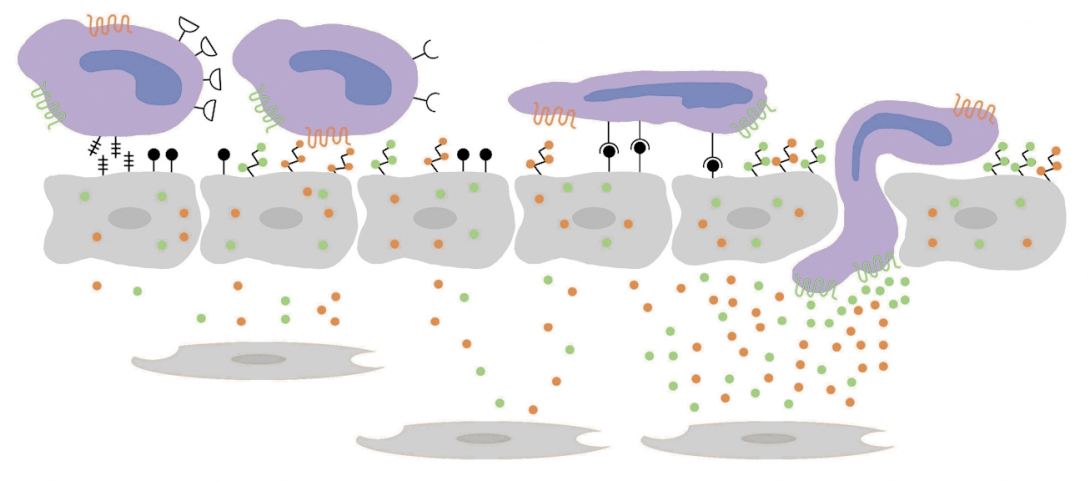

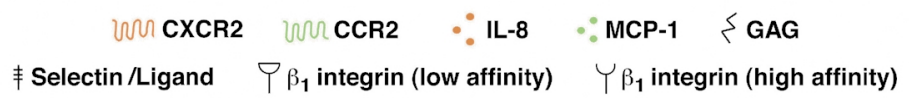

\section{Figure 1}

Chemokine receptors divide the labor of inflammatory infiltration. A monocyte engaged in selectinmediated rolling on vascular endothelial cells is depicted as expressing CXCR2 (red) and CCR2 (green). First, interaction of CXCR2 with its ligand IL-8 (red dots attached to glycosaminoglycans (GAG)) leads to up-regulation of $\alpha_{4} \beta_{1}$ integrin affinity and firm adhesion. Then, interaction of CCR2 with its ligand MCP-1 (green dots attached to GAG) leads to migratory behavior i.e., diapedesis and entry into the subendothelium. Here, chemokines are shown as being presented to cells in the vascular lumen in the context of endothelial cell surface GAGs.

model, MCP-1 and CCR2 should have compensated for the loss of CXCR2 in the chimeras. Insight into why this did not happen comes from work of Huo et al. (15) in this issue of the JCI. These authors examined monocyte interactions with the endothelium of ex vivo perfused carotid arteries from a murine atherosclerosis model. First, they demonstrated that firm adhesion of monocytes to carotid endothelium was inhibited by pertussis toxin, suggesting the involvement of chemokine receptors which are $\mathrm{G}_{\mathrm{\alpha i}}$-linked. Then, the authors showed that at least two chemokines were displayed on the endothelial surface, namely MCP-1 and KC. The latter protein is the closest murine ortholog of the human chemokine GRO- $\alpha$. Mice have no clearcut IL-8 ortholog, although several known murine proteins are significantly homologous to it. Of these, KC appears to be the closest equivalent to IL-8, as judged by its pattern of expression and putative function. KC activates the murine receptor for ELR-containing CXC chemokines, which has, primarily for the sake of convenience, been denoted CXCR2.

The surprise came when Huo et al. (15) attempted to block monocyte adhesion by interfering with specific chemokines. Despite the wealth of 2 must not be involved in adhe sion, but only in migration. In other words, the chemokine system has evolved a strict division of labor and the model of chemokine involvement in atherosclerosis must be expanded to accommodate it (see Figure 1).

\section{Specialization and the profusion of chemokines}

Besides demonstrating that CXCR2 can be a therapeutic target in athero- sclerosis, these results also have important biological implications. One of the enduring puzzles in the chemokine field is the large number of ligands and receptors that seem to have overlapping functions. On the ligand side, there are a dozen chemokines that attract neutrophils, another dozen that attract monocytes, and another dozen that attract $T$ cells. On the receptor side, a single cell can express several chemokine receptors. Why should that be the case if a chemokine receptor is simply needed to get a cell from here to there? It appears now that these homologous proteins have different functions. We have known for some time that ELRcontaining CXC chemokines that bind to CXCR2 with similar affinities actually elicit different responses from neutrophils $(11,19)$. For example, some are more efficient chemoattractants while others are more efficient inducers of the respiratory burst.

The data from Huo et al. (15) suggest that an analogous division of labor may exist among receptors. Perhaps monocytes require both CXCR2 and CCR2 because the former is more efficiently connected to the adhesion apparatus while the latter communicates more effectively with transmigration machinery. Rather than try to make one receptor do both things, nature has taken a page from The Wealth of Nations in order to increase the efficiency of inflammatory cell infiltration. In the case of atherosclerosis, of course, efficiency is not to be celebrated since it only enhances disease progression. However, the primary function of these cells is to protect us from foreign invaders, and in this context efficient responses should confer a great selective advantage. It will be interesting to see if, in other cases, the specialization of chemokines and chemokine receptors does indeed enhance the opulence of host protection.
1. Rossi, D., and Zlotnik, A. 2000. The biology of chemokines and their receptors. Annu. Rev. Immunol. 18:217-242.

2. Libby, P. 2000. Coronary artery injury and the biology of atherosclerosis: inflammation, thrombosis, and stabilization. Am. J. Cardiol. 86:3-8.

3. Rollins, B. 2000. MCP-1, -2, -3, -4, -5. In Cytokine reference. J. Oppenheim et al., editors. Academic Press. London, United Kingdom. 1145-1160.

4. Gu, L., et al. 1998. Absence of monocyte chemoattractant protein-1 reduces atherosclerosis in low density lipoprotein receptor-deficient mice. $\mathrm{Mol}$. Cell. 2:275-281. 
5. Boring, L., Gosling, J., Cleary, M., and Charo, I.F. 1998. Decreased lesion formation in CCR2 $2^{-/}$mice reveals a role for chemokines in the initiation of atherosclerosis. Nature. 394:894-897.

6. Gosling, J., et al. 1999. MCP-1 deficiency reduces susceptibility to atherosclerosis in mice that overexpress human apolipoprotein B. J. Clin. Invest. 103:773-778.

7. Dawson, T.C., Kuziel, W.A., Osahar, T.A., and Maeda, N. 1999. Absence of CC chemokine receptor-2 reduces atherosclerosis in apolipoprotein Edeficient mice. Atherosclerosis. 143:205-211.

8. Boisvert, W.A., Santiago, R., Curtiss, L.K., and Terkeltaub, R.A. 1998. A leukocyte homologue of the IL-8 receptor CXCR-2 mediates the accumulation of macrophages in atherosclerotic lesions of LDL receptor-deficient mice. J. Clin. Invest. 101:353-363.

9. Moser, B., et al. 1993. Expression of transcripts for two interleukin 8 receptors in human phagocytes, lymphocytes and melanoma cells. Biochem. J. 294:285-292.
10. Chuntharapai, A., Lee, J., Hebert, C.A., and Kim, K.J. 1994. Monoclonal antibodies detect different distribution patterns of IL-8 receptor A and IL-8 receptor B on human peripheral blood leukocytes. J. Immunol. 153:5682-5688.

11. Walz, A., Meloni, F., Clark-Lewis, I., von Tscharner, V., and Baggiolini, M. 1991. [Ca2+]i changes and respiratory burst in human neutrophils and monocytes induced by NAP-1/interleukin-8, NAP-2, and gro/MGSA. J. Leukoc. Biol. 50:279-286.

12. Lundahl, J., Skold, C.M., Hallden, G., Hallgren, M and Eklund, A. 1996. Monocyte and neutrophi adhesion to matrix proteins is selectively enhanced in the presence of inflammatory mediators. Scand. J. Immunol. 44:143-149.

13. Schwartz, D., et al. 1994. Role of the GRO family of chemokines in monocyte adhesion to MMLDL-stimulated endothelium. J. Clin. Invest. 94:1968-1973.

14. Gerszten, R.E., et al. 1999. MCP-1 and IL-8 trigger firm adhesion of monocytes to vascular endothelium under flow conditions. Nature. 398:718-723.
15. Huo, Y., et al. 2001. The chemokine KC, but not monocyte chemoattractant protein-1, triggers monocyte arrest on early atherosclerotic endothelium. J. Clin. Invest. 108:1307-1314.

16. Gong, J.H., and Clark-Lewis, I. 1995. Antagonists of monocyte chemoattractant protein 1 identified by modification of functionally critical $\mathrm{NH}_{2}$-terminal residues. J. Exp. Med. 181:631-640.

17. Zhang, Y.J., Rutledge, B.J., and Rollins, B.J. 1994. Structure/activity analysis of human monocyte chemoattractant protein-1 (MCP-1) by mutagenesis: identification of a mutated protein that inhibits MCP-1-mediated monocyte chemotaxis. J. Biol. Chem. 269:15918-15924.

18. Ni, W., et al. 2001. New anti-monocyte chemoattractant protein-1 gene therapy attenuates atherosclerosis in apolipoprotein E-knockout Mice. Circulation. 103:2096-2101.

19. Moser, B., Clark-Lewis, I., Zwahlen, R., and Baggiolini, M. 1990. Neutrophil-activating properties of the melanoma growth-stimulating activity, MGSA. J. Exp. Med. 171:1797-1802. 